\title{
Escritos biomédicos de dermatología en la Revista Médica Hondureña
}

\author{
Dermatology biomedical papers in the Revista Médica Hondureña
}

\author{
Nelly Janeth Sandoval Aguilar
}

Dermatóloga, Sub-especialista en Dermatopatología; Departamento de Patología, Hospital Escuela; Departamento de Medicina Interna, Facultad de Ciencias Médicas UNAH; Tegucigalpa.

RESUMEN. La dermatología como especialidad en Honduras tuvo sus inicios en los años 50 , con los aportes imperecederos de los pioneros Eduardo Fernando Selva, Hernán Corrales Padilla y Héctor Laínez. En una revisión de la Revista Médica Hondureña (RMH), en el periodo comprendido entre 1930 a marzo 2020, se identificaron 93 artículos publicados por dermatólogos hondureños. La primera publicación fue en 1952 por el Dr. Corrales Padilla, cuyo legado en la dermatología sobrepasó las fronteras de Honduras; publicó más de 56 artículos en revistas nacionales e internacionales entre los años 50 hasta su fallecimiento en 1999, de los cuales 16 fueron en la RMH. En este artículo se presentan antecedentes históricos relevantes y los aportes de la dermatología hondureña a través de la RMH. Se revisaron diferentes fuentes de información (Biblioteca Virtual en Salud Honduras, base de datos Literatura Latinoamericana en Ciencias de la Salud, documentos impresos). Palabras clave: Cuidados de la piel, Dermatología, Honduras, Publicaciones.
Recibido: 15-09-2020; Aceptado para publicación 18-10-2020

Dirección para correspondencia: Dra. Nelly J. Sandoval,

Correo electrónico: nelly.sandoval@unah.edu.hn

Declaración de relaciones y actividades financieras y no financieras y conflictos de interés: ninguna.

DOI: https://doi.org/10.5377/rmh.v88iSupl.1.11677

\section{INTRODUCCIÓN}

La primera escuela de dermatología se instaló en el Hospital Saint Louis de París en 1801. Honduras tuvo sus primeros dermatólogos más de un siglo después, en la década de los 50 , con los doctores Eduardo Fernando Selva (1924-1969), Hernán Corrales Padilla (1924-1999) y Héctor Laínez (1930-1996). Estos médicos tuvieron una visión científica y académica la cual está plasmada en sus publicaciones, destacando el doctor Corrales Padilla con la mayor cantidad de trabajos científicos. La producción científica del Dr. Corrales Padilla consta de más de 56 artículos en revistas nacionales e internacionales, entre la década de los 50 hasta su fallecimiento en $1999 .{ }^{1}$ El objetivo del presente escrito es describir los antecedentes históricos relevantes y los aportes de la Dermatología hondureña a través de la Revista Médica Hondureña, desde sus pioneros hasta la actualidad. Se realizó una búsqueda en la Biblioteca Virtual en Salud Honduras (BVS-HN), en la base de datos Literatura Latinoamericana en Ciencias de la Salud (LILACS, https://lilacs. bvsalud.org/es/) del Centro Latinoamericano y del Caribe de Información en Ciencias de la Salud (BIREME) y memorias de congresos científicos.

\section{LA DERMATOLOGÍA EN HONDURAS}

Desde sus comienzos podemos encontrar evidencia de las contribuciones académicas que han marcado la historia de la Dermatología en Honduras y Centroamérica; por ejemplo, los doctores Eduardo Selva y Corrales Padilla en su afán por ampliar las fronteras del conocimiento, acudieron a la convocatoria organizada por los Dermatólogos de El Salvador, al que sería el primerCongreso Centroamericano de Dermatología, celebrado del 5 al 8 de diciembre de 1957. Al evento asistieron 17 dermatólogos centroamericanos, quienes se convertirían en socios fundadores de la Sociedad Centroamericana de Dermatología. ${ }^{2}$ A partir de esa fecha, quedó establecido como tradición, la realización de este evento cada 2 años, rotando la sede en cada país miembro. Honduras sería sede del tercer Congreso Centroamericano de Dermatología, del 6 al 10 de diciembre de 1961 en Tegucigalpa. ${ }^{2}$ Para coordinar la organización del primer evento científico de tal envergadura en esta área de la medicina, los Doctores Eduardo Selva, Hernán Corrales Padilla y Héctor Laínez, fundaron el 13 
de febrero de 1960 la Sociedad Hondureña de Dermatología, ${ }^{1}$ y alcanzaron el cometido con gran éxito.Hasta la fecha se han celebrado 31 congresos, el último del 23 al 27 de octubre de 2019 en la Ciudad de San Salvador, El Salvador. La próxima sede sería en Honduras, en noviembre del 2021, pero por la pandemia de COVID-19, se cambió tentativamente para noviembre de 2022, si las condiciones sanitarias y económicas lo permiten.

Entre las décadas del 50, 60 y 70 llegaron a ser 14 los profesionales en esta rama de la medicina en Honduras, todos formados en el extranjero. Sin embargo, a partir de la década de los 80 este patrón cambió, gracias a la visión y gestión principal del Dr. Corrales Padilla, con la apertura en 1984 del programa de Postgrado de Dermatología en la Universidad Nacional Autónoma de Honduras (UNAH). ${ }^{1}$ Desde sus inicios, este programa de residencia médica ha mantenido un alto grado de compromiso científico y académico, gracias a destacados profesores como el Dr. Hernán Corrales Padilla, Dr. Héctor Laínez, Dra. Virginia Figueroa, Dra. Nohemí Álvarez y Dra. Ofelia Wilkinson, quienes formaron a los profesionales que hoy se dedican a continuar el proceso (Figuras 1 y 2). A la fecha existen 115 dermatólogos en el país, de los cuales 53 (46.1\%) son egresados de este postgrado.

Esta visión científica y académica de los precursores, ha llevado a los dermatólogos a destacarse como escritores de publicaciones científicas. En una revisión de la Revista Médica Hondureña en la Biblioteca Virtual en Salud de Honduras y en la base de datos LILACS-BIREME, en el período de 1930 a marzo de 2020, se encontraron 93 artículos publicados por dermatólogos hondureños en la que participan como autores 0 coautores. La primera publicación realizada por un dermatólogo hondureño, según esta revisión, fue en 1952, por el Dr. Corrales Padilla, ${ }^{3.5}$ quién de los 56 artículos publicados en revistas nacionales e internacionales, $16(28.6 \%)$ están publicados en la $\mathrm{RMH}^{3-18}$ El Dr. Corrales Padilla es el dermatólogo hondureño con mayor número de publicaciones en dicha revista, siguiéndole en orden de frecuencia los colegas, Dr. Héctor Laínez Núñez $15,{ }^{14,15,18,19-30}$, Dra. Nelly Sandoval $11,{ }^{1,31-40}$ Dr. Elmer López Lutz $10,{ }^{41-50}$ y Dr. Gustavo Lizardo $10 .{ }^{50-59}$ Como dato relevante de las nuevas generaciones podemos mencionar que en el Volumen 76, No. 3 de 2008, 9 de los 11 artículos que constituyen este número, fueron escritos por dermatólogos. . $^{1,28,39,51,60-64}$

En cuanto a los tipos de artículos publicados, el más común es el artículo tipo caso clínico 21 (22.6\%), 3,6,18,21,30,31,39,50,52,53,55,56,65${ }^{73}$ que es una de las estrategias didácticas y muy atractiva en la enseñanza-aprendizaje en todas las ramas de la medicina y su publicación sirve de apoyo en la práctica clínica de los médicos. Siguen en frecuencia los artículos originales $19(20.4 \%),{ }^{4,7,12-}$ 15,17,44,47,48,51,74-81 6 de ellos producto de trabajos de tesis de graduandos del Postgrado de Dermatología UNAH. 17,44,47,48,51,76 Es de resaltar también que varios de los trabajos de tesis han sido galardonados como mejor trabajo científico de los Postgrados de la UNAH, obteniendo el tercer lugar en 1996 (Dra. Benilda Martel Ramos), primer lugar en 1998 (Dr. Rafael Valle Pinto), primer lugar en 2004 (Dra. Miriam González Matute y Dra. Verónica Meléndez Oviedo), ${ }^{51}$ segundo lugar en 2015 (Dra. Carolina López Chiessa, Dra. Clelian Espinal Castañeda y Dra.

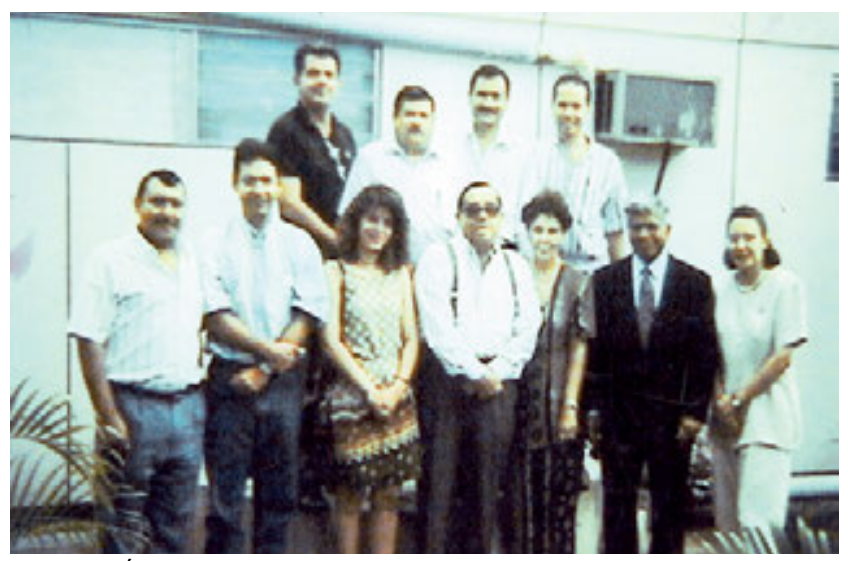

Figura 1. Última promoción del Postgrado de Dermatología con "el Maestro", año 1996. De izquierda a derecha; Atrás: Dr. Ángel Cruz, Dr. José Morales, Dr. Gustavo Lizardo, "Dr. Elmer López; Adelante: **Dr. Marco Amaya QDDG,

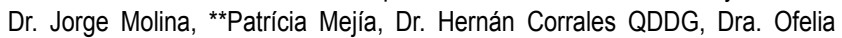
Wilkinson, Dr. Madhu Pathak, ${ }^{*}$ Dra. Benilda Martel. "Residente de 3er Año, ${ }^{* *}$ Residente 2do Año.

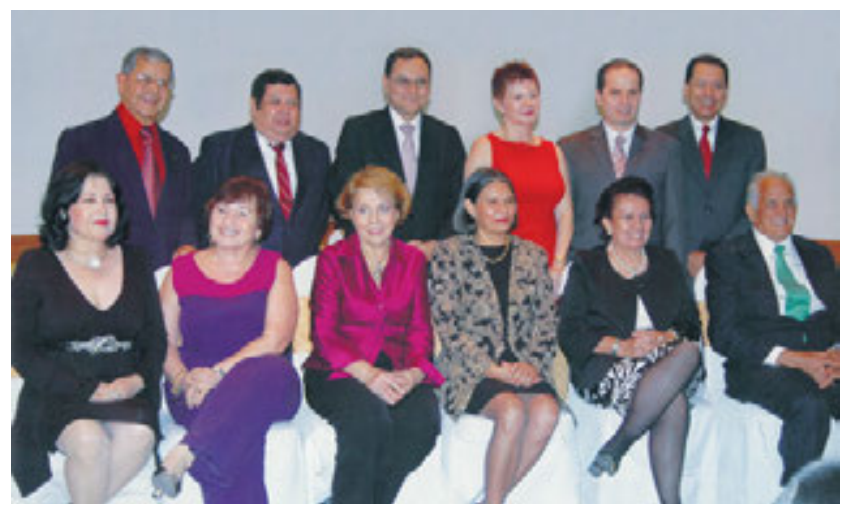

Figura 2. Homenaje a la trayectoria de Dermatólogos de Honduras, Sociedad Hondureña de Dermatología y Cirugía Dermatológica, año 2009. De izquierda a derecha, de pie: Dr. Manuel Leiva, Dr. José Silverio Morales, Dr. Gustavo Lizardo, Dra. Nelly Paz, Dr. Elmer López, Dr. Jorge Molina; sentados: Dra. Alicia Mejía, Dra. Virginia Figueroa QDDG, Dra. Ofelia Wilkinson, Dra. Norma Flores, Dra. Nohemy Álvarez, Dr. Elías Handal QDDG.

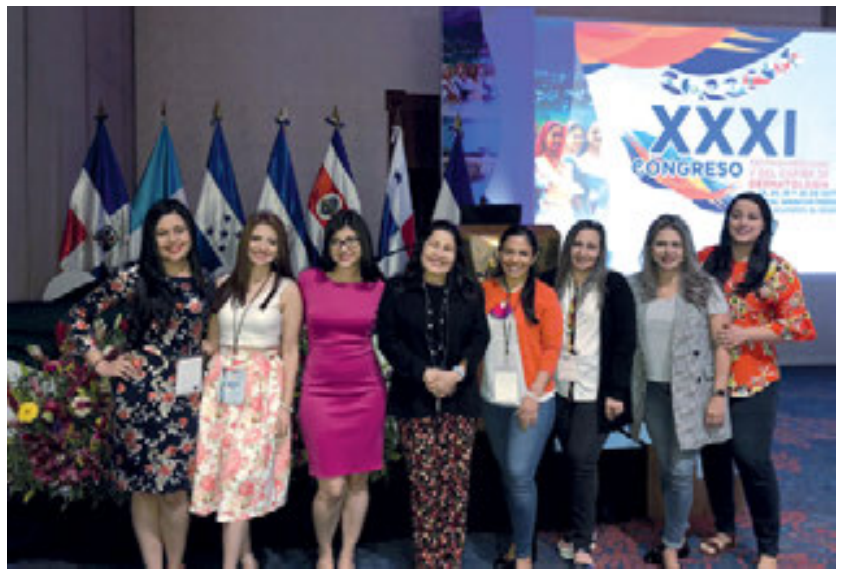

Figura 3. San Salvador, El Salvador, XXXI Congreso Centroamericano y del Caribe de Dermatología, año 2019. La fotografía fue posterior a la presentación del trabajo de investigación. De izquierda a derecha, "Dra. Katia Aguilar, ${ }^{* *}$ Dra. Andrea Enamorado, ${ }^{* *}$ Dra. Gina Guillén, Dra. Alicia Mejía, Dra. Nelly Sandoval, Dra. Nora Escoto, Dra. Xenia Velásquez, Dra. Kathya Chinchilla; *Residente de 3er Año, **Residente 2do Año. 
Johana Carrasco Quintero) y en el Foro de Residentes que se lleva a cabo en el marco de los Congresos Centroamericanos, han sido premiados con el primer lugar en 2002 (Dr. Omar Zúniga Mendoza), 2004 (Dra. Miriam González Matute y Dra. Verónica Meléndez Oviedo), ${ }^{51}$ y 2019 (Dra. Katia Aguilar Gutiérrez y Dra. Aleydi Gómez Campos) (Figura 3). El tercer lugar de los tipos de publicaciones, lo ocupan las revisiones bibliográficas $17(18.2 \%), 5,7-11,16,19,20,32,38,40,43,61,82-84 \mathrm{y}$ casi en igual proporción, las imágenes clínicas $16(17.2 \%), 33,41,42,54,57,62,85-93$ seguido de ensayo/poesía/ad libitum $7(7.5 \%),{ }^{22-28}$ editoriales 5 $(5.4 \%),{ }^{29,34-37}$ historia de la medicina $4(4.3 \%),{ }^{1,45,46,94} \mathrm{y}$ de opinión $4(4.3 \%) .49,63,64,95$

Cuadro 1. Publicaciones de dermatología de acuerdo al tema, Revista Médica Hondureña, 1930-2020, n=93.

\begin{tabular}{lc}
\hline TEMA & $\mathbf{N}(\%)$ \\
\hline Micosis Superficiales y Profundas & $11(11.8)$ \\
Historia /Enseñanza & $11(11.8)$ \\
Enfermedades Parasitarias & $8(8.6)$ \\
Enfermedades Bacterianas & $8(8.6)$ \\
Ensayo/Poesía/Ad Libitum & $7(7.5)$ \\
Cáncer No Melanoma & $5(5.4)$ \\
Eritematoescamosas: Psoriasis & $4(4.3)$ \\
Fotodermatosis & $4(4.3)$ \\
Genodermatosis & $3(3.2)$ \\
Colagenopatías & $3(3.2)$ \\
Melanoma & $3(3.2)$ \\
Terapéutica & $3(3.2)$ \\
Enfermedad Vascular & $2(2.2)$ \\
Enfermedad Metabólica & $2(2.2)$ \\
Tumores Benignos & $2(2.2)$ \\
Liquen Plano & $2(2.2)$ \\
Misceláneas & $15(16.1)$ \\
\hline
\end{tabular}

Los temas abordados se detallan en el Cuadro 1. Destacan en primer lugar, las enfermedades infecciosas micóticas, bacterianas y parasitarias $27(29.0 \%)$, con predominio de las micóticas 11 (11.8\%), describiéndose 6 superficiales, ${ }^{32,38,55,57,67,76}$ 4 profundas, ${ }^{6-8,53}$ y 1 sistémica, ${ }^{87}$ que por ser Honduras un país de clima tropical son patologías muy frecuentes en el medio. Siguen en frecuencia, el tema de historia de la medicina en general, la dermatología y la enseñanza en Honduras, que viene a remarcar la visión legada.

El tema del momento y futuras investigaciones y publicaciones, sin duda es la pandemia de COVID19, impensable para la mayoría de nosotros, pero nos ha permitido ser partícipes del surgimiento de una enfermedad, en el que cada uno de los médicos en sus diferentes ramas, en todas las regiones del mundo, ha tenido que aplicar sus conocimientos para aprender su comportamiento y Dermatología no se ha quedado atrás. Se ha visto que el SARS-CoV-2 es capaz de producir manifestaciones tan variadas en la piel, que se le ha llegado a nombrar como la "nueva gran simuladora", desplazando a la sífilis, con lo cual tendremos un buen cimiento de futuras publicaciones.

\section{CONCLUSIÓN}

Comparado a los dos siglos de existencia en el mundo, la Dermatología en Honduras es una especialidad relativamente nueva que ha crecido en profesionales y en recursos exponencialmente en las últimas décadas. Es importante dejar documentada la contribución de la Dermatología a la historia de la medicina en Honduras y su contribución al quehacer científico en general. Así como hemos aprendido de aquellos que nos precedieron, esperamos que las nuevas generaciones sigan aprendiendo y contribuyendo con publicaciones científicas en esta prestigiosa revista.

\section{REFERENCIAS}

1. Sandoval NJ. Precursores y pioneros de la dermatología en Honduras. Rev Med Hondur. 2008;76(3):132-137.

2. Sociedad Centroamericana y del Caribe de Dermatología. Historia y congresos celebrados [Internet]. Santo Domingo: SCCD; 2011. [Consultado el 28 de Julio de 2020]. Disponible en: www.sccad.net

3. Corrales Padilla H. Panvasculoneuritis. Rev Med Hondur. 1952;20(5):294297.

4. Alcerro JN, Corrales Padilla H, Cueva Adán. Escleroma rino-faringo-laringeo en Honduras. Rev Med Hondur. 1952;20(5):275-283.

5. Corrales Padila H. Síndrome post-trombótico de los miembros inferiores. Rev Med Hondur. 1952;20(5):244-260.

6. Corrales Padilla H. Micosis profundas. Rev Med Hondur. 1953;21(1):311331.

7. Corrales Padilla H. Micosis profunda en Honduras. Rev Med Hondur. 1955;23:(4).

8. Corrales Padilla H. Cromomicosis. Rev Med Hondur. 1970;38(2): 48-54.

9. Corrales Padilla H. Fotodermatosis. Rev Med Hondur. 1973;41(2):104108.

10. Corrales Padilla H. Queilitis por fotosensibilidad. Rev Med Hondur. 1975;43(3):163-177.

11. Corrales Padilla H. Avances recientes en trastornos Dermatológicos inducidos por fotosensibilidad a drogas. Rev Med Hondur. 1979;47(4):117-130.
12. Corrales Padilla H. Ethambutol e isoniacida en el tratamiento de la tuberculosis. Rev Med Hondur. 1970;38(1):3-8.

13. Corrales Padilla H, Laínez Núñez H. Pacheco J. Calagualina. Su ensayo en el tratamiento de la psoriasis. Rev Med Hondur. 1972;40(4):253-260.

14. Corrales Padilla, H. Fotoquimioterapia de la Psoriasis con Psoralenos por vía oral y luz negra. Rev Med Hondur. 1975;44(2):96-105.

15. Corrales Padilla $H$, Cerellino NA, Laínez Núñez H, Espinoza V, de Sierra Ofelia, Alvarez N, et al. Doble ciego comparando anapsos versus placebo en el tratamiento de psoriasis. Rev Med Hondur. 1984;52(2):90-92.

16. Corrales Padilla H, Corrales Álvarez H, Carías S. Asociación entre "hla" y enfermedad, estado actual y perspectivas. Rev Med Hondur. 1985;53(2):104-118.

17. Martel $\mathrm{Bl}$, Corrales Padilla $\mathrm{H}$, Ponce $\mathrm{C}$, de Ponce E. Criocirugía como alternativa terapéutica en leishmanisis cutánea atípica. Rev Med Hondur 1997;65(2):62-64.

18. Corrales Padilla $\mathrm{H}$. Laínez Núñez $\mathrm{H}$. Leishmaniasis cutánea diseminada (revisión del tema e informe del primer caso en Honduras). Rev Med Hondur. 1968;36(2):142-148.

19. Laínez Núñez $H$. Inmunobiología en trasplante de órganos y tejidos. Rev Med Hondur. 1970;38(1):9-11.

20. Laínez Núñez H. Las Colagenosis: Lupus eritematoso, dermatomiositis, esclerodermia, escleredema. Rev Med Hondur. 1960;28:(3). 
21. Laínez Núñez H, Fernández Selva E. Forma aguda de la enfermedad de chagas, importancia semiológica del signo de Romana. Rev Med Hondur 1971;39:5-15.

22. Laínez Núñez $\mathrm{H}$. Canto a la Infancia ¿Qué significa a mi entender un niño? Rev Med Hondur. 1968;36(4):356-359.

23. Laínez Núñez H. Aseguran que somos. un país de contrastes. Rev Med Hondur. 1971;39(1)84-85.

24. Laínez Núñez H. Niños pobres de América. Rev Med Hondur. 1971;39(3):342--343.

25. Laínez Núñez H. "Minamata y Márcala" Un pasado aprendido y una lección olvidada. Rev Med Hondur. 1973;41(1):4-13.

26. Laínez Núñez H. Angustia en el rancho. Rev Med Hondur. 1973;41:(1).

27. Laínez Núñez H. Credo médico. Rev Med Hondur. 1984;52:(3).

28. Laínez Núñez H. Amor de madre. Rev Med Hondur. 2008;76(3):138.

29. Laínez Núñez H. El Hospital General de Tegucigalpa, fiel reflejo de una crisis Hospitalaria a nivel nacional 1970. Rev Med Hondur. 1970;38(4)170173.

30. Durón RA, Laínez Núñez H. Enfermedad de Mondor. tromboflebitis superficial de la pared anterior del tórax) informe de los primeros casos en Honduras. Rev Med Hondur. 1967;35(4):216-223.

31. Sandoval Aguilar NJ, Martel Ramos B, Prudot VP. Leiomiosarcoma cutáneo primario desarrollado sobre cicatriz de quemadura. Rev Med Hondur. 2007;75(3):129-132.

32. Sandoval NJ, Arenas R, Giussiano G, García D, Chávez, Zúniga P Diagnóstico y tratamiento de dermatofitosis y pitiriasis versicolor. 2012;80(2):6674.

33. Sandoval NJ, Velásquez X. Melanoma maligno amelanótico. Rev Med Hondur. 2008;76(2):64.

34. Sandoval NJ. La revista médica hondureña a la vanguardia de la investigación científica. Rev Med Hondur. 2011;79(2):57.

35. Sandoval NJ. El tabaco fuente de divisas, pobreza y enfermedad. Rev Med Hondur 2011;79(4):181-182.

36. Sandoval NJ. Comités éticos asistenciales. Rev Med Hondur. 2012;80(2):46.

37. Sandoval NJ. Parasitosis intestinal en países en desarrollo. Rev Med Hondur. 2012;80(3):89.

38. Espinoza CG, Sandoval NJ, González M. Pitiriasis versícolor. Rev Med Hondur 2008;76(1):28-32.

39. González M, Ortiz Paredes K, Sandoval NJ. Lupus Eritematoso Cutáneo Subagudo Reporte de un caso y Revisión de la Literatura. Rev Med Hondur 2008;76(3):114-117.

40. Medina E, Carbajal B, Ponce C, Sandoval N, Valladares E. Las Porfirias. Rev Med Hondur. 2000;68(1):16-24.

41. López Lutz EA. Linfogranuloma Venéreo. Rev Med Hondur. 2000;68(4):15

42. López Lutz EA. Lepra Lepromatosa Difusa en Reacción Lepromatosa. Rev Med Hondur. 2000;68(2):60.

43. López Lutz EA, Cruz Banegas A. Psoriasis, una enfermedad sistémica con un tratamiento limitado. Rev Med Hondur 2013;81(2-4):121-124.

44. Lemus Rosa LL, Montes Guifarro J, López Lutz E, Erazo Trimarchi G. Acantosis nigricans en estudiantes de medicina de la UNAH. Rev Med Hondur. 2008;76(2):60-63.

45. Bú Figueroa E, Alger J, López Lutz E. Dr. Carlos Javier A. Zepeda Premio de Educación Médica Contínua Dr. Jorge Haddad Quiñónez, año 2003. Rev Med Hondur. 2003;72(3):160-162.

46. Alger J, Alvarenga RE, López Lutz E, Maradiaga E, Rivera O, Robles Elba, et al. Reseña histórica del Congreso Médico Nacional y presentación del XLVI Congreso, Tegucigalpa 2003. Rev Med Hondur. 2003;71(1):48-54.

47. Espinoza C, Matute N, Ochoa Rueda S, López Lutz E. Caracterización clínica de pacientes con síndrome de ovario Poliquístico. Rev Med Hondur. 2011;79(3):128-131.

48. Matute N, Espinoza C, Alger J, Padgett D, López Lutz E, Zúniga C. Caracterización clínico - epidemiológica de pacientes con leishmaniasis atendidos en el Hospital Escuela. Rev Med Hondur. 2009;77(1):7-15.

49. Alger J, Rivera Vega O, Maradiaga E, Aguilera EG, Alvarenga RE, Duarte RM, et al. XLVI Congreso Médico Nacional Dr. Pablo J. Cambar. Rev Med Hondur 2003;71:156-164.

50. Durón RM, Lizardo G, López Lutz E, Morales S, Hesse H, Molina L, et al.
Síndromes neurocutáneos en la consulta neurológica. Serie de dasos. Rev Med Hondur. 2009;77(4):172-176.

51. Lizardo Castro GA, Ponce C. Ponce E, Sierra M. Caracterización de pacientes con leishmaniasis cutánea atípica. Rev Med Hondur. 2008;76(3):101107.

52. Lizardo Castro GA, García D. ¿Qué debemos saber hoy en día de la fascitis necrotizante? A Propósito de dos casos. Rev Med Hondur. 2011;79(4):203207.

53. Lizardo Castro GA, Zúniga Muñoz P. Esporotricosis linfocutánea en niños: A propósito de un caso. Rev Med Hondur. 2011;79(2):81.

54. Lizardo-Castro GA, García DM. Liquen estriado. Rev Med Hondur. 2011;79(2):74.

55. Lizardo Castro GA, Lizardo AE. Presentación inusual de onicomicosis por Candida Albicans. Rev Med Hondur. 2012;80(2):61-65.

56. Lizardo Castro GA, Velásquez DG, Cruz Enamorado B. Hemangioma congénito rápidamente involutivo. Rev Med Hondur. 2015;83(3y4):130-133.

57. Lizardo-Castro GA, Velásquez DG. Candidiasis miliar por pañal. Rev Med Hondur. 2015;83(1 y 2):55.

58. Lizardo Castro G, Gómez Campos A. Larva migrans cutánea: casos pediátricos. Rev Med Hondur 2019;87(2):84.

59. Lizardo Castro GA, Gómez Campos A. Larva Migrans cutánea: casos pediátricos. Rev Med Hondur. 2019;77(3):84.

60. González M, Zambrano M. Siringomas Eruptivos Reporte de un Caso y Revisión de la Literatura. Rev Med Hondur. 2008;76(3):118-120.

61. Morales S, Matute N. Lo que el Médico General debe saber sobre Escabiosis. Rev Med Hondur 2008;76(3):121-127.

62. Morales S, Matute N. Carcinoma basocelular ulcerado. Rev Med Hondur 2008;76(3):113.

63. Mejía Al, Wilkinson O. La enseñanza de la dermatología en el pregrado en la UNAH. Rev Med Hondur. 2008;76(3):128-129.

64. Espinoza Figueroa D. La Dermatología en Honduras: una especialidad joven pero con mucha historia. Rev Med Hondur. 2008:76(3):130-131.

65. Molina JA, Javier Zepeda CA. Infección Secundaria a mordedura de gato. Rev Med Hondur 1997;65(4):120-123.

66. Godoy Romero A. Respuesta excelente a una aplicación de nitrógeno líquido en una melanosis de labio. Rev Med Hondur. 2000;68(2):66-68.

67. Godoy Romero A. Calap Calatayud J, García Martos P. Onicomicosis causada por Microsporum Racemosum: Primer Caso descrito en el mundo. Rev Med Hondur. 2000;68(2):69-71.

68. Godoy Romero A, Arévalo Rosado F, Calap Calatayud J. Canicie ciliar circunscrita adquirida. Rev Med Hondur. 2000;68(3):110-113.

69. Mejía P. Sarcoma de Kaposi Clásico Presentación de un caso y revisión. Rev Med Hondur 2000;68(1):27-29.

70. Hernández M, Lemus L, Bú Figueroa E. Manifestación inusual de fiebre tifoidea. Presentación de un caso y revisión de literature. Rev Med Hondur. 2004;72(2):97-99.

71. Matute N, Meléndez V. Síndrome de ectrodactilia-displasia ectodérmicalabio/paladar hendido. Hondur. 2007;75(1):26-29.

72. Ortíz Paredes K, Meléndez V. Atrofodermia de pasini y pierini unilateral. Rev Med Hondur. 2010;78(2):80-82.

73. Escoto N, García D. Acné Fulminans presentación de un caso. Rev Med Hondur. 2011;79(3):133-135.

74. Molina Rodríguez JA, Javier Zepeda CA. Resistencia del gonococo a la penicilina. Informe preliminar de un estudio en Tegucigalpa. Rev Med Hondur. 1979;(47):43-45.

75. Handal AE. Tratamiento de las dermatosis mediante productos populares. Rev Med Hondur. 1982;(50):88-90.

76. Mejía Al. Micosis superficiales en el Hospital Escuela. Rev Med Hondur. 1991;59:8-13.

77. Grispan S, Garda S, Molina S. Donación voluntaria de sangre y derivados en el IHSS: un modelo de auto-abastecimiento institucional. Rev Med Hondur. 1991;63(3):118-122.

78. Fernández Vásquez JA, Cadillo F, Pérez G, Cruz Banes A, Menjívar A, Laínez $V$. Análisis de los problemas en la enseñanza de la semiología médica en la FCM de la UNAH. Rev Med Hondur. 1995;63(2):52-56.

79. Sánchez A, Barahona LM. Experiencia clínica con el uso de interleucina-3. Rev Med Hondur. 1995;63(4):129-134. 
80. Alvarado T, Molina J, Cruz R, Rivera E, Herrera J, Peña E, et al. Estudio clínico-epidemiológico de las aguas de consumo humano de la Villa de Cofradía, Cortés y su influencia en la población. Rev Med Hondur. 2006;74:174-187.

81. Bustillo-Ponce AJ, Hernández Palma MA, Ramírez Rivera DM, Madrid Rubí MW. Estilos de vida no saludables en estudiantes de zona urbanorural, la Esperanza-Intibucá, Honduras, 2012-2013. Rev Med Hondur. 2013;81(2-4):82.

82. Handal AE. Introducción al estudio de las genodermatosis. Rev Med Hondur. 1967;35:241-239-242.

83. Mejía Al. Liposucción, principios, técnicas y resultados. Rev Med Hondur. 1996;64(1):13-18.

84. Wilkinson Oberti O, Velásquez Montoya X, Herrera M. Melanoma maligno: cuando sospecharlo y que hacer. Rev Med Hondur. 2002;70(2):86-90.

85. Bermúdez Lacayo J, González M. Melanoma maligno. Rev Med Hondur. 2003;71(4):199.

86. Bermúdez Lacayo J, Cruz Perdomo EJ, Bermúdez Lacayo S. Metástasis cutánea. Rev Med Hondur. 2004;72(2):138.

87. Bermúdez Lacayo J, Bermúdez Arriola BI. Mucormicosis. Rev Med Hondur. 2005;73(4):166.

88. González M, García D. Prurigo Nodular. Rev Med Hondur. 2011;79(3):132.

89. González M, Zúniga M. Liquen plano oral. Rev Med Hondur. 2011;79(4): 197.

90. González M. Carcinoma de células escamosas asociado a cromoblastomicosis. Rev Med Hondur. 2013;81(1):30.

91. Guevara DE, Vijil M, Valladares I, Chinchilla K. Siameses onfalópagos. Rev Med Hondur. 2011;79(2):73.

92. Martínez- Rodríguez P, Oliva Cáceres L. Flujometría doppler en medicina materno fetal. Rev Med Hondur. 2014;82(1):27-32.
93. Matute N. Xantomas eruptivos en paciente con diabetes mellitus y dislipidemia mixta. Rev Med Hondur. 2013;81(2-4):106.

94. Martel BI, Bú Figueroa E. Grandes figuras de la medicina hondureña: El Doctor Hernán Corrales Padilla 1996. Rev Med Hondur. 1996;64(3):116117.

95. Morales S. 15 años de experiencia en la prevención del cáncer de piel. Rev Med Hondur. 2011;79(4):214-215.

ABSTRACT. Dermatology as a specialty in Honduras had its beginnings in the $50 \mathrm{~s}$, with the enduring contributions of the pioneers Eduardo Fernando Selva, Hernán Corrales Padilla and Héctor Laínez. A review of the Revista Médica Hondureña (RMH), from 1930 to March 2020, identified 93 articles published by Honduran dermatologists. The first publication was in 1952 by Dr. Corrales Padilla, whose legacy in dermatology crossed the borders of Honduras; he published more than 56 articles in national and international journals between the 1950s until his death in 1999, of which 16 were in the RMH. This article presents relevant historical backgrounds and Honduran dermatology contributions through $\mathrm{RMH}$. Different sources of information were reviewed (Virtual Library in Health Honduras, Latin American Literature in Health Sciences data base, printed documents).

Keywords: Dermatology, Honduras, Publications, Skin care. 